\title{
Can the Cardiopulmonary 6-Minute Walk Test Reproduce the Usual Activities of Patients with Heart Failure?
}

\author{
Guilherme Veiga Guimarães, Giovanni Bellotti, Fernando Bacal, Amilcar Mocelin, \\ Edimar Alcides Bocchi \\ São Paulo, SP - Brazil
}

Objective - The 6-minute walk test is an way of assessing exercise capacity and predicting survival in heart failure. The 6-minute walk test was suggested to be similar to that of daily activities. We investigated the effect of motivation during the 6-minute walk test in heart failure.

Methods - We studied 12 males, age $45 \pm 12$ years, ejection fraction $23 \pm 7 \%$, and functional class III. Patients underwent the following tests: maximal cardiopulmonary exercise test on the treadmill (max), cardiopulmonary 6minute walk test with the walking rhythm maintained between relatively easy and slightly tiring (levels 11 and 13 on the Borg scale) (6EB), and cardiopulmonary 6-minute walk test using the usual recommendations ( $6 R U)$. The $6 E B$ and $6 R U$ tests were performed on a treadmill with zero inclination and control of the velocity by the patient.

Results - The values obtained in the max, $6 E B$, and 6RU tests were, respectively, as follows: $O_{2}$ consumption $\left(\mathrm{ml} \mathrm{kg}^{-1} \cdot \mathrm{min}^{-1}\right) 15.4 \pm 1.8,9.8 \pm 1.9(60 \pm 10 \%)$, and $13.3 \pm 2.2$

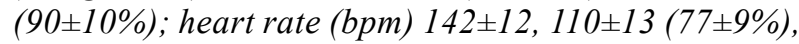

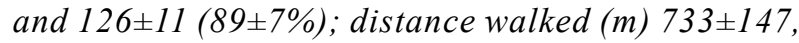
$332 \pm 66$, and $470 \pm 48$; and respiratory exchange ratio $(R)$ $1.13 \pm 0.06,0.9 \pm 0.06$, and $1.06 \pm 0.12$. Significant differences were observed in the values of the variables cited between the max and $6 E B$ tests, the max and $6 R U$ tests, and the $6 E B$ and $6 R U$ tests $(p<0.05)$.

Conclusion-Patients, who undergo the cardiopulmonary 6-minute walk test and are motivated to walk as much as they possibly can, usually walk almost to their maximum capacity, which maynotcorrespond to that of their daily activities. The use of the Borg scale during the cardiopulmonary 6-minute walk test seems to better correspond to the metabolic demand of the usual activities in this group of patients.

Key words: heart failure, exercise, oxygen consumption

Instituto do Coração do Hospital das Clínicas - FMUSP

Mailing address: Guilherme Veiga Guimarães - Rua Dr. Baeta Neves, 98 - 05444-050

São Paulo, SP, Brazil - E-mail: gvguima@usp.br

English version by Stela Maris C. e Gandour
A limited tolerance for effort is frequently the first and major clinical characteristic of heart failure, reflecting a reduction in cardiac function and alteration in peripheral response ${ }^{1,2}$. The cardiopulmonary test is used to assess the functional capacity, therapeutic response, and prognosis in this group of patients ${ }^{3,4}$. However, some patients may have difficulty in undergoing the maximum cardiopulmonary exercise test, and limitation to maximum exercise does not reflect the usual activity ${ }^{5}$.

The 6-minute walk test is a simple method for assessing exercise capacity and predicting survival in patients with heart failure ${ }^{6-8}$. The type of exertion perceived during a 6-minute walk test has been suggested to be similar to that of daily activities ${ }^{3,9}$. In addition, the patient may determine the walking rhythm, which is an additional advantage for the more physically limited patient, who certainly would not tolerate the maximal exercise test ${ }^{10}$. However, the walking intensity during the 6 minutes may be influenced by verbal encouragement ${ }^{11}$.

The Borg scale is a descriptive marker of subjective physical exertion, whose degree of intensity corresponds to an odd number, categorized at 15 degrees varying from 6 to $20^{12}$. Quantification of the exertion perceived and the heart rate is linearly related to the intensity of the physical activity ${ }^{13,14}$. In addition to being a valid and reliable indicator of exercise intensity, the Borg scale is reproducible and may be used in repetitive tests ${ }^{15}$.

The objective of our study was to compare the effect of motivation on the results of the cardiopulmonary 6-minute walk test in patients with heart failure using the Borg scale (between 11 and 13) and the usual recommendation about the intensity of exertion.

\section{Methods}

From March to April 1997, we consecutively studied 12 patients with heart failure, which in 10 was due to idiopathic dilated cardiomyopathy and in 2 was due to ischemic cardiomyopathy, who were hospitalized to undergo assess- 
ment for surgical or clinical treatment for heart failure. All patients were males in New York Heart Association (NYHA) functional class III.

The following patients were excluded from the study: patients whose medicamentous therapy had not been optimized within 1 month, patients with orthopedic problems or with noncardiovascular limitations to exertion, with atrial fibrillation, or cachexia, those interrupting the walking test prior to the $6^{\text {th }}$ minute, and those whose respiratory exchange ratio $\left[\mathrm{R}\left(\mathrm{VCO}_{2} / \mathrm{VO}_{2}\right)\right]$ in the maximal cardiopulmonary exercise test did not reach a value $>1.05^{16}$. Female patients were not included in the study due to the reduced number of females assessed during the study period.

All patients with heart failure referred for cardiopulmonary assessment during the period studied were hospitalized in the clinical sector of the Heart Failure and Transplantation Unit of InCor of the Hospital das Clínicas of the FMUSP. These patients had been previously advised about the technique and protocol of the study. All patients were studied in a temperature-controlled environment $\left(21-23^{\circ} \mathrm{C}\right)$ at least 2 hours after a meal, and they were using their medication (digitalis, diuretics, angiotensin-converting enzyme inhibitors). All assessments were part of the clinical procedures and were performed after the patients agreed that the results could be reported in the scientific literature.

The maximal cardiopulmonary exercise test, the 6-minute walk test, using the Borg scale, and usual recommendations for exertion intensity were randomized, conducted by the same professional, and performed on 3 consecutive days at the same hour.

Initially, all patients underwent resting standard 12lead electrocardiography and exercise testing with continuous electrocardiographic monitoring (Max 1; Marquette Electronics; Milwaukee, WI, USA), with blood pressure monitoring through the auscultation method, and with monitoring of ventilation and gas exchange during the exercise test. The test was performed on a programmable treadmill (Series 2000; Marquette Electronics; Milwaukee, WI, USA) according to the modified Naugthon protocol ${ }^{17}$. After 2 minutes standing without exercising, all patients were encouraged to exercise until the symptoms (fatigue or dyspnea) prevented them from continuing the test. The ventilatory data of oxygen consumption and carbon dioxide production were obtained at each respiratory cycle using the computerized system (Vmax 229 model, SensorMedics, Yorba Linda, CA, USA), and the analysis of the data collected was performed through the arithmetic mean of intervals at each 60 seconds. Peak oxygen consumption $\left(\mathrm{VO}_{2}\right.$ peak) was considered the highest $\mathrm{VO}_{2}$ reached during exercise ${ }^{18}$, which was used as an index of maximal exercise capacity for each individual.

The cardiopulmonary 6-minute walk test using the Borg scale was performed on a treadmill with zero inclination and velocity controlled by the patient. All patients were advised to maintain walking velocity during the test between relatively easy and slightly tiring (between 11 and 13 on the Borg scale) ${ }^{10}$. The following 3 alert phrases were standardized for the patients to adjust their walking rhythm between 11 and 13: "if it is easy, increase the velocity," "if it is between 11 and 13, maintain the velocity," and "if it is above 13, reduce the velocity." The cardiopulmonary variables were measured during the test, and the values found in the last 60 seconds were considered the maximal values. The distance walked during the 6 minutes was recorded by the microprocessor of the manual control of the treadmill.

The cardiopulmonary 6-minute walk test according to the usual recommendations was performed on a treadmill with zero inclination, and the patient had control of the velocity ${ }^{19}$. During the test, patients were systematically encouraged to increase velocity to walk the most they could. Encouragement was standardized with phrases like "if you can walk faster, increase the velocity," "you are doing very well," and "if it is very tiring, you can reduce the velocity." Blood pressure was measured at rest and at the $6^{\text {th }}$ minute. The electrocardiographic, ventilatory, and gas exchange variables were continuously assessed during the test. The mean values of the last 60 seconds of the test were considered the maximum cardiopulmonary variables. The microprocessor of the manual control of the treadmill recorded the distance walked during the test.

The descriptive analysis was presented as mean and standard deviation. The variables studied underwent the analysis of variance (ANOVA), and the statistical significance level adopted was $p<0.05$. When significance was found, the post-hoc analysis of Bonferroni was performed.

\section{Results}

All patients completed the 3 tests with no complications.

In the maximal cardiopulmonary exercise test, the mean value of the maximum heart rate (HRmax) was $142 \pm 12 \mathrm{bpm}$, of the peak oxygen consumption $\left(\mathrm{VO}_{2}\right.$ peak) was $15.4 \pm 1.8$ $\mathrm{ml} \cdot \mathrm{kg}^{-1} \cdot \mathrm{min}^{-1}$, and of the maximum distance was $733 \pm 147 \mathrm{~m}$. All patients reached levels considered as maximum exertion $\mathrm{R}$ (respiratory exchange ratio) $>1.05(1.13 \pm 0.06)$.

In the cardiopulmonary 6-minute walk test using the Borg scale, the mean distance walked was $332 \pm 66 \mathrm{~m}$ ( $45 \%$ of the maximum distance), the mean $\mathrm{VO}_{2}$ was $9.8 \pm 1.9 \mathrm{ml} . \mathrm{kg}$ ${ }^{1} \cdot \mathrm{min}^{-1}\left(60 \%\right.$ of the $\mathrm{VO}_{2}$ peak $)$, the mean heart rate was $110 \pm 13$ bpm ( $77 \%$ of the HRmax), and R was $0.90 \pm 0.04$ ( $79 \%$ of the maximumR).

In the cardiopulmonary 6-minute walk test using the usual recommendations, the mean distance walked was $470 \pm 48 \mathrm{~m}$ ( $64 \%$ of the maximum distance), the mean $\mathrm{VO}_{2}$ was $13.3 \pm 2.2 \mathrm{ml} . \mathrm{kg}^{-1} \cdot \mathrm{min}^{-1}\left(90 \%\right.$ of the $\mathrm{VO}_{2}$ peak $)$, the mean heart rate was $126 \pm 11 \mathrm{bpm}(89 \%$ of the HRmax), and R was $1.06 \pm$ 0.05 ( $94 \%$ of the maximumR).

When comparing the results of the tests, the distance walked was statistically different in the different tests (fig. 1). Oxygen consumption (fig. 2), heart rate (fig. 3), and the respiratory exchange ratio (fig. 4) also showed significant differences in the 3 assessments. 


\section{Discussion}

Our results showed that motivation during exercise may have determined better physical performance, such as an increase in the distance walked, in oxygen consumption, and in heart rate. Assessment of submaximal exercise capacity on the treadmill with zero inclination and velocity controlled by the patient was also possible in heart failure.

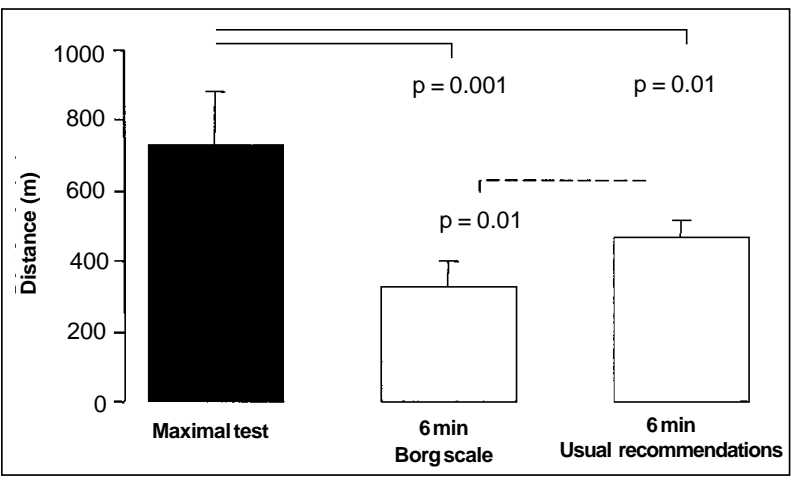

Fig. 1 - Distance walked in the maximal cardiopulmonary exercise test, in the 6 minute walk test using the Borg scale with relatively easy and slightly tiring rhythms (11 and 13), and in the 6-minute walk test with the usual recommendations.

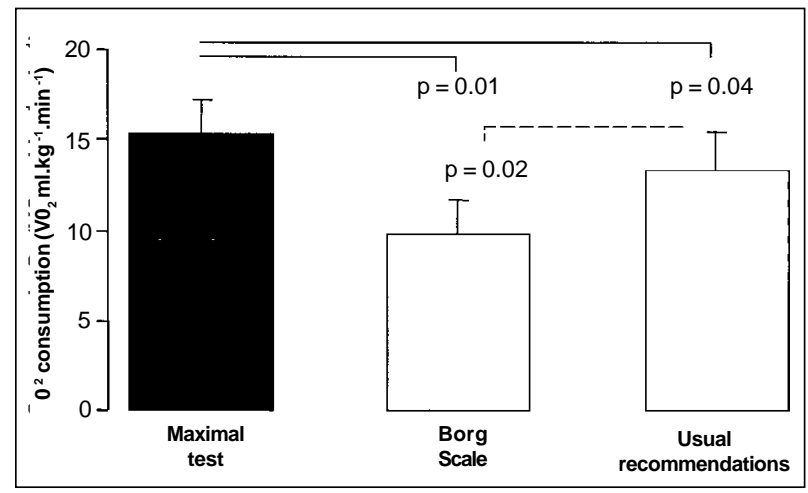

Fig. 2 - Oxygen consumption in the maximal cardiopulmonary exercise test, in the 6 minute walk test using the Borg scale with relatively easy and slightly tiring rhythms (11 and 13), and in the 6-minute walk test with the usual recommendations.

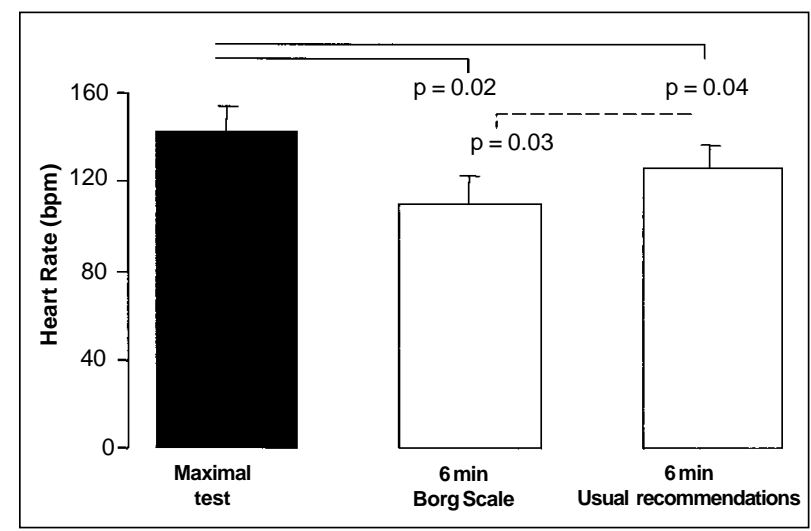

Fig. 3-Heart rate in the maximal cardiopulmonary exercise test, in the 6-minute walk test using the Borg scale with relatively easy and slightly tiring rhythms (11 and 13 ), and in the 6-minute walk test with the usual recommendations.

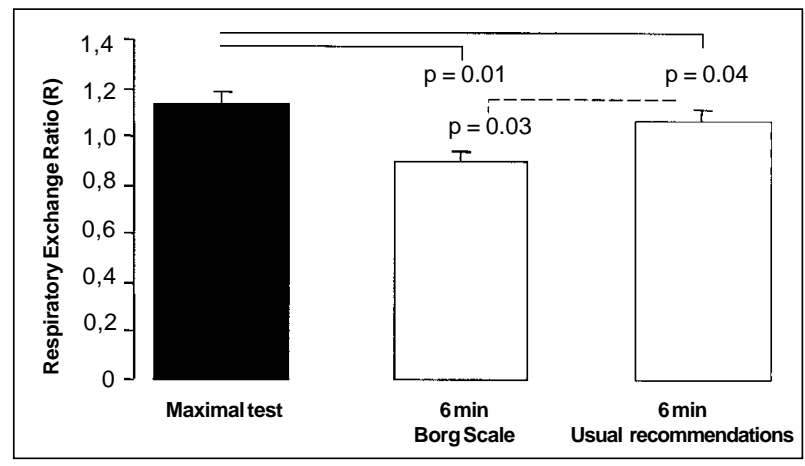

Fig. 4-Respiratory exchange ratio in the maximal cardiopulmonary exercise test, in the 6-minute walk test using the Borg scale with relatively easy and slightly tiring rhythms (11 and 13), and in the 6-minute walk test with the usual recommendations.

Performance of the cardiopulmonary 6-minute walk test on a treadmill proved to be safe and that patients could collaborate in the testing by controlling velocity.

The physiological alterations induced by exertion depend essentially on the intensity of the overload. During exercise, systolic volume increases from rest to mild to moderate exercises, maximal values being achieved between $40 \%$ to $50 \%$ of $\mathrm{VO}_{2}$ peak. From these values on, cardiac output increases more due to a higher heart rate ${ }^{20,21}$. We demonstrated that, when encouraged to walk as fast as possible, patients walked at $\mathrm{VO}_{2}$ and heart rate intensities close to the maximum ( $90 \%$ and $89 \%$, respectively). When advised to walk at intensities between relatively easy and slightly tiring (between 11 and 13 in the Borg scale), their walking intensity was $60 \%$ of $\mathrm{VO}_{2}$ peak and $77 \%$ of the maximum heart rate. This fact confirms that which has already been demonstrated, that the adequate pacing rhythm of an individual is around 60 to $70 \%$ of $\mathrm{VO}_{2}$ peak $^{22}$. In addition, exercise intensity may be classified according to physiological stress, ie, from 30 to $74 \%$ of $\mathrm{VO}_{2}$ peak and from 35 to $79 \%$ of the maximum heart rate, which are activities considered mild to moderate; above those levels, the activities are considered intense ${ }^{18}$.

The Borg scale has a good correlation with some physiological variables. A 0.85 correlation was demonstrated in relation to oxygen consumption, heart rate, pulmonary ventilation, and lactate levels. In this regard, the scale may provide objective data of the degree of fatigue during the test. The Borg scale may be useful to assess constancy versus change in the individual exertion during the 6 minutes of the test ${ }^{23}$. Therefore, the aerobic capacity and performance during the 6-minute walk test may be higher or lower, and with the walking rhythm maintained between relatively easy and slightly tiring (11 and 13), this may reflect the physical condition or clinical condition, and not higher or lower work for the patients.

The increase in the distance walked due to verbal motivation influenced the performance of the patients in the cardiopulmonary 6-minute walk tests. The means of the group were, respectively, $470 \mathrm{~m}$ and $332 \mathrm{~m}$ when encouraged to walk as fast as possible and when advised to walk between a relatively easy and slightly tiring rhythm accor- 
ding to the Borg scale. As has already been reported by other authors ${ }^{4-6}$ and shown in our study, the level of encouragement may have a crucial effect on the individual's performance. The distance walked during the 6-minute test is a marker of worse prognosis and poorer clinical condition in patients with functional class II and III heart failure ${ }^{3}$. Patients with better performance were shown to have a lower mortality rate and to require less hospitalization due to heart failure and other causes ${ }^{3,7}$. According to the distance walked, the patients were divided into levels as follows: level 1 - patients who walked less than $300 \mathrm{~m}$; level 2 - patients who walked between 300 and $375 \mathrm{~m}$; level 3 - those who walked between 375 and $450 \mathrm{~m}$; and level 4 - those who walked more than $450 \mathrm{~m}$. The result of our study showed that when the patients were encouraged to walk as fast as possible, they reached level 4 . However, when advised to walk in a rhythm between relatively easy and slightly tiring, they reached level 2.

The cardiopulmonary 6-minute walk test with velocity controlled by the patient requires maximal patient's collaboration. However, the number of patients studied was limited. All patients were in functional class III, hospitalized, and compensated. The randomization of the tests may not have had the training effect as has already been reported ${ }^{18,24}$.

This study shows that the 6-minute walk test, when applied in its original form, achieves relatively high percentages of exertion, suggesting that the determinants of the submaximal and maximal exercise capacity in this group of patients may not differ. Considering that one of the objectives of the 6-minute test is to reproduce the daily activities of the patients with heart failure, the use of walking rhythms between 11 and 13 on the Borg scale during the walking test seems more appropriate for this outcome.

In conclusion, our results are the first, in our environment, to show that when patients undergoing the cardiopulmonary 6-minute walk test are encouraged to walk as fast as they possibly can, they walk close to their maximum, and this may not correspond to their daily activities. The use of the Borg scale during the cardiopulmonary 6-minute walk test seems to better correspond to the metabolic demand of the usual activities in this group of patients. We suggest that the Borg scale should be used between the relatively easy and slightly tiring rhythm (11 and 13) during the 6-minute walk test to assess the usual activities in this group of patients.

\section{References}

1. Drexier H, Riede U, Munzel T, et al. Alterations of skeletal muscle in chronic heart failure. Circulation 1992; 85: 1751

2. Wilson JR, Mancini DM. Factors contributing to the exercise limitation of heart failure. J Am Coll Cardiol 1993; 22: 93A-8A.

3. Szlachcic J, Massie BM, Kramer BL, et al. Correlates and prognostic implication of exercise capacity in chronic congestive heart failure. Am J Cardiol 1985; 55: 1037-42.

4. Fleg JL, Piña IL, Balady G, et al. Assessment of functional capacity in clinical and research applications. Circulation 2000; 102: 1591-7.

5. Lucas C, Stevenson LW, Johnson W, et al. The 6-min walk and peak oxygen consumption in advanced heart failure: aerobic capacity and survival. Am Heart J 1999; 138: 618-24.

6. Bittner V, Weiner DH, Yusuf S, et al. Prediction of mortality and morbidity with a 6 minutes walk test in-patients with advanced heart failure. JAMA 1996; 270 : 1702-7.

7. Lipkin DP, Scriven AJ, Crake T, Poole-Wilson PA. Six minute walking test for assessing exercise capacity in chronic cardiac failure. Br Med J 1998; 292: 653-5.

8. Dracup K, Walden JA, Stevenson LW, Brecht ML. Quality of life in patients with advanced heart failure. J Heart Lung Transplant 1992; 11: 273-9.

9. Oliveira MT, Guimarães GV, Barretto AC. Teste de seis minutos em insuficiência cardíaca. Arq Bras Cardiol 1998; 31:387C.

10. Hendrican MC, McKeelvie RS, Smith T, et al. Functional capacity in patients with congestive heart failure. J Card Fail 2000; 6: 214-9.

11. Guyatt G, Pugsley S, Sullivan MJ, et al. Effect of encouragement on walking test performance. Thorax 1984; 39: 818-22.

12. Borg G. Psychophysical bases of perceived exertion. Med Sci Sports Exerc 1982; 14: 377-81.

13. Pandolf KB. Differentiated ratings of perceived exertion during physical exercise. Med Sci Sports Exerc 1982; 14: 397-405.
14. Skinner JS, Hutsler R, Bergsteinova V, et al. Perception of effort during differents types of exercise and under different environmental conditions. Med Sci Sports 1973; 5: 110-5.

15. Skinner JS, Hutsler R, Bergsteinova V, et al. The validity and reliability of a rating scale of perceived exertion. Med Sci Sports 1973; 5: 94-6.

16. Katz SD, Berkowitz R, LeJemtel TH. Anaerobic threshold detection in patients with congestive heart failure. Am J Cardiol 1992; 15: 1565-69.

17. Patterson J, Naughton J, Pietras RJ. Treadmill exercise in assessment of functional capacity of patients with severe left ventricular disease. Am J Cardiol 1972; 30: $757-62$.

18. Wasserman K, Hausen JE, Sue DY, Whipp BJ, Casaburi R. Principes of Exercise Testing and Interpretation. $2^{\text {nd }}$ ed. Philadelphia: Lea \& Febiger, 1994.

19. Guyatt GH, Sullivan MJ, Thompson PJ, et al. The 6-minute walk: a new measure of exercise capacity in patients with chronic heart failure. Can Med Assoc J 1985; 132: 919-23.

20. Romano M, Monteforte I, Cardei S, et al. Cardiopulmonary exercise response in patients with left ventricular dysfunction or heart failure: noinvasive study by gas exchange and impedance cardiography monitoring. Cardiology 1996; 87 : 147-52.

21. Koike A, Itoh H, Taniguchi K, et al. Detecting abnormalities in left ventricular function during exercise by respiratory measurement. Circulation 1989; 80: 1737-46.

22. Fletcher GF, Balady G, Froelicher VF, et al. Exersice standards: a statement for healthcare professionals from the American Heart Association. Circulation 1995; 91: 580-615.

23. Fitts SS, Guthrie MR. Six-minute walk by people with chronic renal failure: assessment of effort by perceived exertion. Am J Phys Med Rehabil 1995; 74: 54-8.

24. Clark AL, Poole-Wilson PA, Coats AJS. Effects of motivation of the patient on indices of exercise capacity in chronic heart failure. Br Heart J 1994; 71: 162-5. 